\title{
Tourisme et vodou en Haïti : nouveaux acteurs et nouvelles dynamiques
}

Tourism and voodoo in Haiti: new actors and new dynamics

Joseph Ronald Dautruche

\section{Q OpenEdition}

\section{Journals}

Édition électronique

URL : http://journals.openedition.org/tourisme/1976

DOI : $10.4000 /$ tourisme. 1976

ISSN : 2492-7503

Éditeur

Éditions touristiques européennes

Référence électronique

Joseph Ronald Dautruche, «Tourisme et vodou en Haïti : nouveaux acteurs et nouvelles dynamiques », Mondes du Tourisme [En ligne], 15 | 2019, mis en ligne le 01 juin 2019, consulté le 30 octobre 2019. URL : http://journals.openedition.org/tourisme/1976 ; DOI : 10.4000/tourisme.1976

Ce document a été généré automatiquement le 30 octobre 2019

\section{cc)}

Mondes du tourisme est mis à disposition selon les termes de la licence Creative Commons Attribution - Pas d'Utilisation Commerciale - Pas de Modification 4.0 International. 


\section{Tourisme et vodou en Haiiti : nouveaux acteurs et nouvelles dynamiques}

Tourism and voodoo in Haiti: new actors and new dynamics

Joseph Ronald Dautruche

\section{Introduction}

1 Ces dernières décennies sont marquées par une synergie croissante entre le tourisme et la culture. Les destinations se sont tournées de plus en plus vers la culture comme un moyen de distinction et la culture est de plus en plus liée au tourisme comme un moyen de générer des revenus et des emplois (Richards, 2001). Selon l'Organisation mondiale $\mathrm{du}$ tourisme (OMT, 2006), le tourisme culturel est l'une des grandes tendances du tourisme mondial et est considéré comme l'un des principaux secteurs de croissance pour l'avenir. L'État haïtien s'inscrit bien dans cette dynamique en présentant le tourisme comme la voie à suivre pour sortir le pays de la stagnation économique et de la pauvreté en misant principalement sur la culture (Ministère de la Planification et de la Coopération externe d'Haïti, 2007 ; Ministère du Tourisme, 2008 ; Jocelyn-Lassègue, 2010).

2 Face à l'emprise du vodou dans le passé touristique d'Haïti ${ }^{1}$ et à l'heure d'une relance $\mathrm{du}$ tourisme dans ce pays, avec la culture comme marqueur principal, il importe d'étudier les nouveaux rapports du couple tourisme/vodou. Il s'agit surtout de rencontrer des pratiquants du vodou, des promoteurs du tourisme ou porteurs de projets, des touristes et de regarder « comment cela se passe» (Lazzarotti, 2011, p. 72) de nos jours.

3 Pour ce faire, nous nous concentrons principalement sur le cas d'un espace de pratique vodou appelé communément Souvenance situé aux Gonaïves (Haïti). Souvenance est l'un des plus anciens et des plus importants lieux de pratiques vodous en Haïti, avec 
Soukri et Badjo, situés également dans la région des Gonaïves. Sa fondation remonterait à 1791, peu après le soulèvement des esclaves dans le Nord. Elle aurait été l'œuvre d'un petit groupe d'esclaves qui s'était réfugié dans la zone pour pratiquer les rites ancestraux. Certains héros de la guerre d'indépendance nationale y auraient participé régulièrement à des rituels vodous. Cet espace est toujours présenté dans les différents projets de développement touristique du pays comme un patrimoine culturel à promouvoir. Il a fait l'objet d'une rénovation en 2009, de la part du Ministère de la Culture et de la Communication d'Haïti. Des festivités vodou se déroulent à Souvenance chaque année, du Vendredi saint au vendredi de la semaine suivante. Nous avons fait une visite exploratoire à Souvenance en 2001, dans le cadre d'une sortie de groupe. Ce premier séjour de deux semaines a donné lieu à un projet de recherche quelques années plus tard. Nous sommes retourné à Souvenance en 2010 pour approfondir le terrain. Durant six mois environ, nous avons réalisé des entretiens avec des interlocuteurs âgés de 40 à 70 ans. Certains vivent en Haïti, d'autres en dehors du pays. Ils nous ont été suggérés par leurs pairs en fonction de leurs intérêts pour le vodou et de leurs implications dans les activités à Souvenance. Nous avons par la suite approfondi certains éléments observés en 2010 au cours d'un nouveau séjour à Souvenance effectué en 2014.

\section{Le couple tourisme/vodou : une relation difficile}

\section{Des rituels et des rites variables}

Les sites religieux, partout dans le monde, de tout temps, ont engendré des déplacements, qu'il s'agisse de participer à une fête religieuse, d'assister à un office ou d'effectuer un pèlerinage afin de rendre hommage à un saint ou une divinité (Chaspoul, 2007). Dans le vodou, cependant, on ne trouve pas de grands temples, de rituels écrits, de prêtres formés à cet effet, un livre sacré donnant des préceptes à suivre ou un livre présentant les différents rituels et leur forme ${ }^{2}$. Dans le vodou, les pratiques varient d'une zone à l'autre ou d'une famille à l'autre; les rituels changent en fonction des ougan (prêtre vodou) et des manbo (prêtresse vodoue), de leurs compétences et de leurs habiletés, ainsi que l'a remarqué Métraux au cours de ses recherches :

Le [vodou] est une religion pratiquée par des confréries autonomes dont chacune a souvent son style et ses traditions propres. Il n'existe pas, quoi qu'on en dise, une doctrine et une liturgie [vodoues] auxquelles prêtres et prêtresses soient tenus de se conformer. (1958, p. 15)

5 Le vodou ne donne pas lieu à des catégorisations clairement délimitées. On peut certes établir des nuances, mais les différences irréductibles entre les rites (congo, rada, nago, petro, etc.) sont apparues comme des points d'attention des différents observateurs interrogés. Le vodou est donc une réalité non homogène et cela peut rendre difficile la compréhension de cette pratique.

\section{Un vodou « rejeté » et banalisé à plusieurs niveaux}

6 Le vodou était considéré comme un " culte démoniaque ", une "superstition barbare " et, en conséquence, quelque chose qui salissait la réputation d'Haïti à l'extérieur, principalement dans la presse étasunienne. Cette pratique religieuse a fait l'objet de plusieurs campagnes d'éradication. Celle conduite par l'Église catholique, avec l'appui 
de l'État haïtien, de 1941 à 1942 a été menée de façon plus systématique, visant non seulement le vodou mais également tout ce qui relevait de l'héritage africain. Au cours de cette campagne, des pratiquants du vodou ont été forcés de prononcer un serment solennel devant un prêtre catholique pour justifier leur renoncement à ce culte considéré comme satanique, d'où le nom de campagne "rejeté ». Nombre de lieux cultuels ont été détruits, des objets, pour la plupart très anciens, vandalisés et beaucoup d'arbres considérés comme gîtes des lwa (esprit du vodou) abattus et jetés au feu.

7 Face à l'intensité de cette campagne d'éradication, l'ethnologue Alfred Métraux a conduit des recherches relevant d'une "ethnologie de l'urgence ${ }^{3}$ qu'il a publiées en 1958 sous le titre : Le vaudou haïtien. Dans cette étude, il a identifié un ensemble de facteurs pouvant contribuer à la disparition des croyances et des pratiques vodoues, tels que : l'éducation du peuple, un service médical organisé dans les zones rurales, la montée du protestantisme, les campagnes antisuperstitieuses, etc. Mais la menace la plus sérieuse, affirme-t-il, réside dans sa commercialisation, favorisée par le développement du tourisme (Métraux, 1958). Ainsi écrit-il :

Alors que ni l'État ni l'Église n'ont réussi à entamer sérieusement l'emprise du [vodou], le tourisme, sous sa forme la plus commerciale, exerce sur lui une action dissolvante extrêmement rapide. Depuis quelques années, grâce aux efforts du gouvernement, Port-au-Prince se transforme en un grand centre touristique. Chaque américain qui débarque dans cette ville n'a qu'un mot à la bouche: « vodou », et qu'un désir, celui de voir ces cérémonies qu'ils imaginent cruelles et orgiaques. Beaucoup de hougan [prêtre vodou] et de mambo [prêtresse vodoue] qui, depuis quelques années déjà, voyaient avec plaisir des groupes d'Américains assister aux cérémonies de leur houmfo [temple vodou] ont répondu avec empressement à la curiosité de la clientèle étrangère. Les houmfo se sont ouverts largement aux touristes. (Métraux, 1958, p. 47)

Métraux a souligné que certains temples vodous se sont transformés en de véritables salles de spectacle et que des prêtres et prêtresses ont même monté des spectacles qui se répètent chaque semaine en vue d'offrir aux visiteurs «le pittoresque dont ils ont soif ».

D'autres auteurs (Goldberg, 1983 ; Bédard, 1988 ; Daniel, 1996), à la suite de Métraux, ont signalé que des «shows vodous " se sont tenus hors des espaces habituels, voire même dans des hôtels. Une demande que l'on peut mettre en relation avec les publicités dont Haïti a fait l'objet à une certaine époque. En effet, Haïti était présenté par les agences touristiques américaines sous le terme de "vodoo-land " (Métraux, 1958, p. 318) ; sans oublier l'image renvoyée par la " peinture naïve haïtienne », dont les artistes (Hector Hyppolite, Philomé Obin, Castera Bazile...) dépeignaient généralement Haïti comme une île fabuleuse où les gens passent leur temps à danser ou à faire des cérémonies vodoues au milieu de fleurs et de plantes exotiques (Antonin, 1976).

Il est important, toutefois, de préciser que l'offre de "spectacles vodous » a été constatée uniquement dans la capitale du pays. Et Métraux a bien fait remarquer que certains pratiquants du vodou ont abandonné les temples qui s'étaient lancés dans l'organisation de cérémonies pour touristes :

Si les houngan et les mambo se sont fait de gros revenus, ils ont chassé de leurs temples les vrais croyants. [...] Cette exploitation éhontée de la religion par ses propres prêtres ne sévit, il est vrai, qu'à Port-au-Prince mais elle finira par ébranler la foi des campagnards qui subissent l'influence de la capitale. (1958) 
11 Ces relations, peu reluisantes, qui ont marqué le couple tourisme/vodou par le passé constituent encore aujourd'hui un sujet de préoccupation pour les chercheurs, certains pratiquants du vodou et les promoteurs du tourisme.

\section{Nouveaux touristes, nouveaux acteurs et nouveaux projets}

\section{Souvenance, entre espace ludique et lieu de quête de sens}

Souvenance rassemble chaque année, à l'occasion des célébrations du temps de Pâques, des foules issues à la fois de la population locale et de l'étranger. Les cérémonies qui se déroulent à Souvenance perpétuent des pratiques de tradition africaine, principalement dahoméenne (de Dahomey, ancien nom du Bénin), transplantées en Amérique. Le respect des rites dahoméens constitue la spécificité de Souvenance par rapport aux autres lieux de pratiques vodous dans le pays.

Les activités vodoues s'y déroulent à longueur d'année, mais les festivités qui ont lieu $\mathrm{du}$ Vendredi saint au vendredi de la semaine suivante sont les plus marquantes. Ces festivités attirent, à côté des pitit kay (les pratiquants attachés à cet espace) vivant en Haïti ou à l'étranger, des touristes proprement dits, des chercheurs, des étudiants, des journalistes, des membres du gouvernement haïtien et des habitants de la zone. Il s'agit d'un ensemble de rituels vodous, où se mêlent prières, chants, danses, sacrifices de bétail, transe et bain de purification.

Les lieux de pratiques vodous constituent des espaces ludiques et offrent des moments d'évasion que nombre de personnes recherchent dans les activités touristiques ordinaires. Nicolas Vonarx l'a souligné en 2011 dans une énumération de ce qu'il définit comme les fonctionnalités du vodou :

(...) Le vodou offre des moments d'amusement lors des festivités. Dans des rituels annuels ou de simples rencontres organisées chez un ougan, les participants habitués, visiteurs d'un soir ou simples badauds, viennent se divertir et rompre avec une monotonie quotidienne riche en embarras. Ils viennent agrémenter leur journée, boire un coup, manger, rire, et faire des rencontres de bon voisinage entrecoupées de quelques déhanchements sur un fond de tambourinage et de mélopées entraînantes. Cette dimension festive du vodou n'est pas négligeable pour de nombreux Haïtiens qui profitent ici de menus plaisirs (...). (2011, p. 14).

Les touristes locaux sont, de fait, plus à même de profiter de ces moments de réjouissances car ils ne nécessitent pas de grandes dépenses. Ainsi, un covoiturage et un sac à dos bien garni peuvent suffire à garantir une fin de semaine d'escapade à quelqu'un venu de Port-au-Prince par exemple. Les sites vodous sont réputés pour leur hospitalité. On y accueille tous les visiteurs avec le même enthousiasme car, comme on dit souvent dans le vodou, tout le monde peut devenir pitit kay.

Pour d'autres visiteurs, leur présence dans les espaces vodous participe d'une autre préoccupation. Certaines personnes justifient leur déplacement d'abord par le désir de connaître et de comprendre le vodou. Mais ce qui se traduit par un simple besoin de connaissance peut constituer aussi une quête de liens plus profonds. Nombre d'entre eux sont liés au vodou à travers un enracinement familial. Certains Haïtiens d'origine viennent en Haïti et visitent particulièrement les lieux de pratique vodous dans 
l'objectif de se rapprocher d'un « chez-soi » originel, pour reprendre l'idée de Caroline Legrand relative aux migrants irlandais (2006, p. 167).

17 Lors d'un entretien ouvert mené à Souvenance en 2010, Rose, une Haïtienne dans la soixantaine vivant en Floride, nous raconte ce qui suit :

Ma mère m'a donné une éducation catholique. Elle est décédée quand j'avais 12 ou 13 ans mais j'ai été membre de l'Église catholique. Mon père, lui, avait l'habitude d'aller à Souvenance à chaque période de Pâques. (...) J'ai grandi et voyagé aux États-Unis d'Amérique sans fréquenter les sites vodous. En 1977, j'ai décidé de retourner au pays et cela a coïncidé avec une période de Pâques. Je me suis dit que je vais profiter pour aller à Souvenance. J'ai demandé à une de mes tantes, qui habitait au centre-ville des Gonaïves, qui était pratiquante du vodou et avait l'habitude de venir à Souvenance, de m'accompagner. J'ai été la bienvenue car le serviteur principal connaissait bien mon père. Depuis, je suis revenue presque à chaque grande cérémonie annuelle et je me suis faite initier en 1985.

J'ai mes fils. Un seul est né en Haïti. Ils ne peuvent pas venir cette année à cause de leurs obligations, mais ils viennent de m'appeler et me dire que l'année prochaine ils seront tous là. (...) Ils rêvent chaque année de venir à Souvenance.

En effet, le souci de connaître une pratique religieuse chérie et valorisée par leurs parents et leurs grands-parents semble motiver les descendants de certains migrants haïtiens de première génération à s'approprier le vodou. Nombre d'entre eux effectuent pour cela un voyage en Haïti chaque année.

Rencontrée à Souvenance en 2010, Claire, une Haïtienne dans la soixantaine vivant à New York depuis environ quarante-sept ans, affirme :

Moi, j'ai mes petits-fils. Ils aiment écouter les musiques vodoues et quand quelqu'un veut changer de musique, ils s'exclament toujours : « it is the music of Mom » [c'est la musique de maman].

Rodrigue, la soixantaine, Haïtien, vit à Montréal depuis les années 1960. Nous l'avons rencontré au cours de notre séjour à Souvenance en 2014. Ses enfants, ne pouvant pas être en Haïti à l'occasion des rituels annuels, essayent de contourner cette nostalgie en demandant à leur père de leur rapporter des objets symboliques issus de ces rituels :

Mes enfants ont l'habitude de venir à Souvenance mais à cause de la situation politique du pays depuis quelques années ils hésitent à y venir. Ils attendent que les choses s'améliorent. Deux d'entre eux ont voulu rentrer cette année mais leur blonde s'est opposée. Ils me demandent toujours d'apporter de l'eau bénite de Souvenance pour eux. (...) C'est très utile. (...) Ils rêvent même de se faire initier au vodou. (...) Quant à moi, je rentre régulièrement au pays, depuis 1987, à l'occasion des festivités à Souvenance.

21 À côté du besoin de connaître le vodou et ses pratiques, il y a visiblement une quête spirituelle personnelle chez certains visiteurs, comme pour Audrey, la cinquantaine, rencontrée à Souvenance en 2010 :

Je suis Martiniquaise. Je suis venue en Haïti pour la première fois en l'année 2000 dans le cadre d'un contrat avec une institution internationale travaillant en Haïti. C'était au mois de novembre. J'ai décidé de venir à Souvenance mais, à ce momentlà, il n'y avait rien. C'est cette année que je suis retournée (2010). J'ai lu pas mal de choses sur le vodou. Je suis à la recherche. Je pense qu'il y a quelque chose d'essentiel dans ces formes de spiritualité. La Martinique a perdu tout ça. Presque tout le monde pratique maintenant les religions européennes. Haïti me fascine beaucoup.

Fabien, un Français dans la trentaine, rencontré à Souvenance en 2010, raconte : 
Je me suis bien renseigné avant de venir en Haïti. Je suis un touriste averti. J'ai lu Métraux, Laënnec Hurbon et les textes de Max Beauvoir, mais j'ai regardé un film aussi. J'ai regardé le film J'ai marché avec un zombi de Jacques Tourneur et ça m'a beaucoup marqué. J'ai voulu venir en Haïti pour rencontrer les communautés pratiquantes et découvrir cette spiritualité. C'est mon deuxième voyage en Haïti. J'ai fréquenté plusieurs sanctuaires vodous. (...) Ce que j'ai regardé dans ce film n'a rien à voir avec le vodou.

Nombre de pratiquants du vodou cherchent, en effet, à changer ces images et s'investissent beaucoup dans ce sens. À l'intérieur comme à l'extérieur du pays, les conférences, expositions, festivals consacrés au vodou, les films-documentaires ne sont pas en reste.

Figure . Des touristes étrangers et des pitit kay autour du bassin Zanmadòn

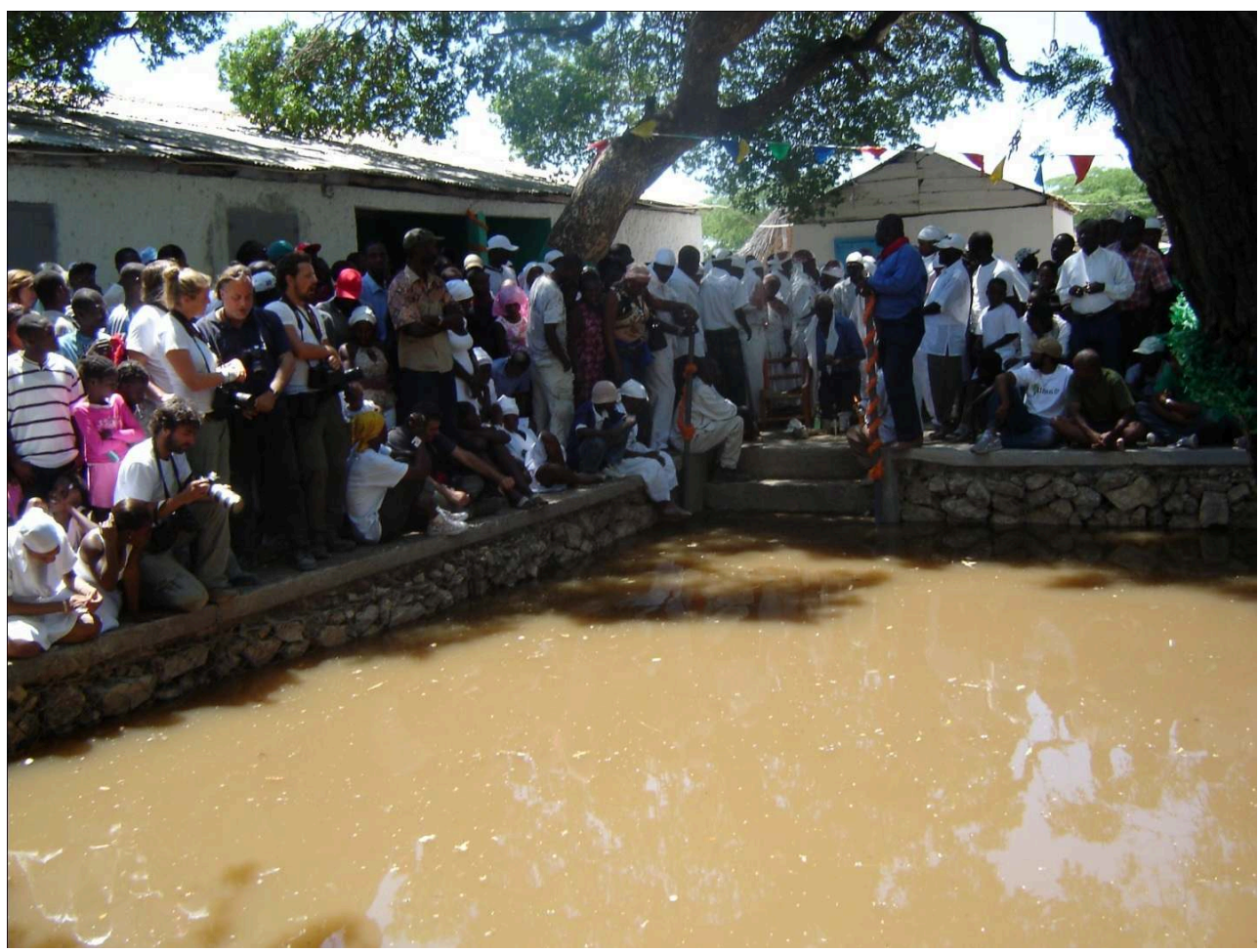

Photo de l'auteur

\section{Faire des Gonaïves une destination « vodou »}

Le territoire gonaïvien abrite les espaces de pratique vodous les plus anciens et les plus connus du pays : Badjo, Soukri et Souvenance. Ces espaces sont souvent présentés dans les plans de développement touristique des différents gouvernements haïtiens comme des éléments du patrimoine culturel à promouvoir. Certains acteurs du vodou envisagent de miser sur ces sites pour faire des Gonaïves une destination touristique avec le label «Gonaïves, capitale du vodou ».

Pour l'un de nos interlocuteurs, rencontré à Souvenance en 2010 et responsable d'une association $^{5}$ nationale vodoue, il est plus intéressant de miser sur les espaces structurés, comme ceux des Gonaïves, qui sont bien ancrés dans leur territoire et qui ont une renommée nationale et internationale. Ainsi, souligne-t-il, on trouve aux Gonaïves des festivités vodoues au cours des quatre saisons de l'année : Soukri en été, 
Banda en automne, Badjo en hiver et Souvenance au printemps. Des pitit kay envisagent non seulement d'améliorer les conditions d'accueil à Souvenance mais aussi de faire de cet espace une destination touristique à part entière.

$J^{J}{ }^{6}$, la cinquantaine, est pratiquant du vodou à Souvenance et cadre de la fonction publique en Haïti. Le projet de Jean ${ }^{7}$ consiste principalement à organiser l'accueil des visiteurs sur les trois principaux espaces vodous des Gonaïves. L'aménagement des aires de stationnement, l'amélioration des conditions d'hébergement et sanitaires et l'accompagnement des visiteurs sont les principaux points sur lesquels il met l'accent. Selon lui, c'est l'État haïtien qui devrait contribuer à la réalisation du projet, car ces espaces font partie du patrimoine culturel du pays. Il pense que l'on devrait privilégier l'hébergement chez l'habitant, c'est-à-dire dans les maisons des pitit kay afin d'éviter une exploitation des festivités par d'autres gens qui n'ont rien à voir avec le vodou et ces espaces. Il a l'habitude d'accompagner personnellement des groupes d'étudiants haïtiens et de visiteurs étrangers sur ces sites, mais il pense que certains jeunes connaissant le vodou devraient être en mesure de le faire également. Il souligne finalement qu'on ne peut pas recevoir de grandes foules dans les espaces de pratiques vodous et que l'on devrait accueillir principalement des chercheurs, des croyants et les personnes qui s'intéressent au vodou de façon particulière.

Le rêve de Christine ${ }^{8}$, la soixantaine, pratiquante du vodou à Souvenance, est de changer l'image du vodou à l'étranger et de modifier la perception des gens sur cette croyance et cette pratique religieuses. Elle a commencé par présenter le vodou, tel qu'il est pratiqué à Souvenance, à ses proches. Découvrant ses talents, elle a étendu son projet et donne désormais des interviews dans les médias et des conférences un peu partout et principalement en Floride, aux États-Unis. Elle organise également des voyages pour des petits groupes voulant visiter Souvenance et établir un contact réel avec le vodou. Elle parle de « contact réel » car, avant d'amener les gens à Souvenance, elle leur fait une présentation générale du vodou puis des spécificités de cet espace à l'aide de supports audio-visuels (musique, photos et vidéos de Souvenance).

Le tourisme lié au vodou constitue, selon Murielle ${ }^{9}$, pratiquante du vodou à Souvenance et cadre retraitée de la fonction publique en Haïti, un espace pour apprendre aux gens tout un pan de la culture haïtienne. Son projet, dit-elle, consiste surtout à partager ses expériences, ses connaissances personnelles et à accompagner les gens intéressés à certains aspects particuliers du vodou : les chants, la musique, la danse, la peinture, etc. Elle a ainsi déjà organisé un séjour pour un groupe composé de douze Cubains s'intéressant au répertoire musical du vodou. À moyen terme, elle envisage de monter un programme de formation pour celles et ceux qui veulent devenir guides professionnels. Pour elle, présenter des croyances et des pratiques si complexes exige des guides bien formés en la matière, maîtrisant plusieurs langues, profondément passionnés et dotés d'un bon sens de la communication. Le projet de former des guides lui est venu à l'idée en suivant un jour, à Souvenance, quelqu'un expliquant un rituel à un groupe composé essentiellement de coopérants et de journalistes étrangers. Elle a pris conscience que cette personne partageait les mêmes discours chargés de préjugés qui sont généralement véhiculés sur le vodou. En quoi une telle interprétation va être utile à un étranger qui a dépensé son argent pour venir en Haïti en vue de comprendre les pratiques du vodou, s'est-elle alors interrogée? Ne va-t-il pas repartir avec les mêmes images figées? Si, au cours d'un second voyage, un étranger peut éventuellement se débrouiller seul, elle doute fort que cela soit possible lors d'un 
premier voyage. Elle croit que le premier contact doit se faire avec l'encadrement d'un guide professionnel. Or même un ougan ou un manbo ou un pratiquant quelconque, qui a tout appris par jeu de rôle, n'a pas forcément la capacité à tenir un discours cohérent face à un touriste, qui exige souvent des explications élaborées sur les croyances et les pratiques vodoues. Le dernier point de son projet est de créer, à long terme, un petit musée mettant en valeur la peinture, les objets, le répertoire musical, les instruments de musique ou un centre d'interprétation de la vie religieuse à Souvenance, lié aux formes d'organisation de l'ancien royaume du Dahomey.

On comprend ici que les porteurs de ces différents projets cherchent à mettre le vodou au goût du jour, à travers de nouveaux récits. Cela s'inscrit dans un combat d'images, à l'instar de celui qui a été mené par les indigénistes haïtiens (avec Jean Price-Mars comme chef de file), dans lequel le vodou, diabolisé, est devenu une revendication identitaire. En effet, l'occupation étasunienne d'Haïti (1915-1934) est venue renforcer le besoin d'opposer une identité essentiellement haïtienne face à l'« étasunisation »du pays. Une " communauté affective ", pour reprendre l'expression d'Appadurai (1996), a alors commencé à se construire. Certains habitants d'Haïti, qui se sentaient «français » ou " américains ", ont été appelés à se définir comme haïtiens. Ce qui a ouvert encore davantage le champ au mouvement indigéniste, qui va embrasser principalement les pratiques culturelles venues d'Afrique jusqu'alors non valorisées. Ce que Carlo Célius appelle " un procès de redéfinition » $(2007$, p. 345$)$ et avec lequel, soutient-il, le vodou est posé comme l'un des principaux pôles identificatoires. C'est dans ce contexte que se déploie l'œuvre de Jean Price-Mars. Dans son ouvrage Ainsi parla l'oncle, paru en 1928, il écrit :

[...] Toutes nos croyances populaires reposent sur des actes authentiques de foi et se concrétisent, en fin de compte, en une religion [le vodou] qui a son culte et ses traditions. (1998, p. 28)

L'œuvre de Jean Price-Mars a profondément inspiré toute une génération d'intellectuels haïtiens et a suscité une série de travaux et d'articles parus principalement dans le Bulletin du bureau d'ethnologie ${ }^{10}$. Price-Mars a le mérite, selon Métraux (1958), d'avoir rendu le vodou respectable et même sympathique ${ }^{11}$ à l'opinion publique haïtienne, en exorcisant le fantôme qu'il était devenu.

31 Afin de sortir du cycle de diabolisation, le projet actuel vise, au-delà des écrits, à esthétiser les espaces de pratiques vodous. En s'appuyant sur le tourisme, l'objectif est de rendre ce "patrimoine difficile $»^{12}$ plus respectable sur le plan national et international.

\section{Le film The oblivion tree, une valorisation du rituel du retour}

Les films participent beaucoup à la construction des destinations touristiques (Vergopoulos et Bourgatte, 2001). Ils constituent des dispositifs de médiation qui mettent en relation des univers narratifs et des territoires ainsi que des pratiques culturelles. Les productions filmiques ont la capacité de promouvoir les environnements (naturels et culturels) dont elles se servent en toile de fond. Elles participent dans ce sens à la qualification ou requalification de certaines pratiques et de certains lieux apparemment banals pour en faire des choses dignes d'un intérêt touristique. Le film-documentaire The oblivion tree, du réalisateur haïtien Norluck Dorange ${ }^{13}$, sorti en Floride en $2009^{14}$, s'inscrit dans cette perspective. 

les captifs exécutaient un rituel au cours duquel ils tournaient autour d'un arbre afin d'oublier leur patrie et leur souffrance. Cet arbre, appelé communément «l'arbre de l'oubli ", n'existe plus aujourd'hui. Il a été remplacé par un autre, le kpatiman en langue Fon-gbé ou l'hysope africana en Botanique, utilisé depuis des siècles au Bénin pour des purifications. À ses côtés, la statue d'une sirène symbolise une destination inconnue pour les Africains déportés (Dorange, 2008, 2009 ; Poda, 2010).

À Souvenance, est exécuté chaque année le « rituel du retour ", qui est en quelque sorte une reprise en sens inverse du rituel qui a été institué en Afrique. Il constitue un retour dans une Afrique originelle, une Afrique mythique.

Figure . Le tour de l'arbre à Souvenance

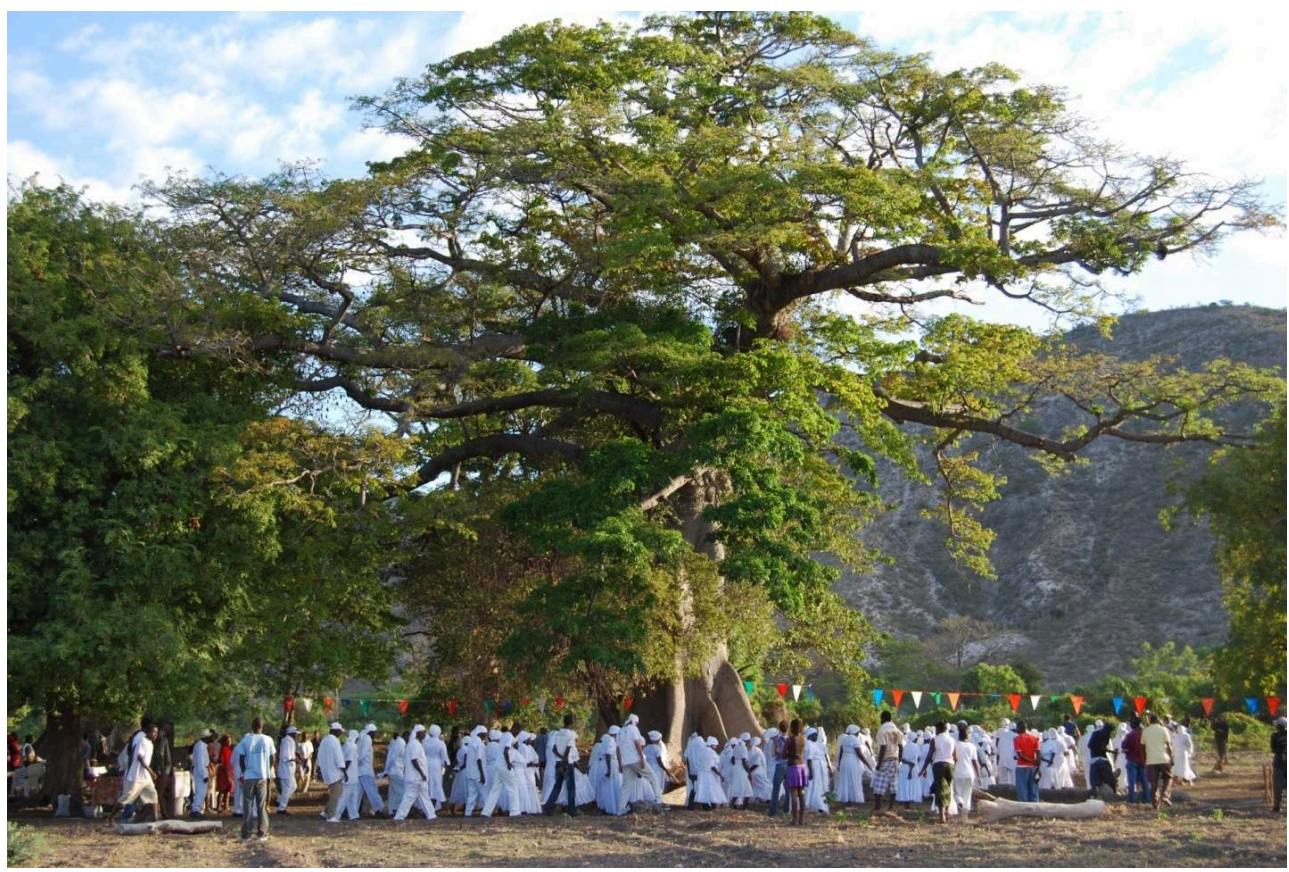

Photo Norluck Dorange, avec la courtoisie de l'auteur

Le site de Souvenance est projeté dans le film-documentaire The oblivion tree de Norluck Dorange comme un lieu servant à soutenir la mémoire africaine en Haïti et " participant activement à sa construction et à sa structuration $»^{15}$. Il met en lumière ce rituel spécifique étroitement lié à la commémoration de la mémoire de l'esclavage et permet aux intéressés de l'apprécier dans son contexte. Il s'inscrit ainsi dans un processus de réagencement des dispositifs de médiation du vodou comme chose à voir (Dautruche, 2013).

\section{Conclusion}

Notre objectif dans cet article était de faire la lumière sur les nouveaux rapports du couple tourisme/vodou. Nous avons observé et rencontré un groupe de pratiquants à Souvenance. Des études que nous avons réalisées sur le carnaval de Jacmel, le Rara de Léogâne (Dautruche, 2011, 2013) et cette recherche nous ont permis d'avancer qu'audelà des préoccupations économiques affichées par les différents gouvernements 
haïtiens, le tourisme est regardé par plusieurs groupes particuliers du pays comme un forum permettant de valoriser leur communauté et de changer l'image de leurs expressions culturelles ou religieuses. Celles-ci varient d'une communauté à l'autre, d'un groupe à l'autre, voire d'une personne à l'autre.

Nous avons également découvert un tourisme pratiqué par des Haïtiens ayant très peu de connaissances du vodou, par des visiteurs étrangers en quête de pratiques religieuses "authentiques » et par des migrants haïtiens de première génération et leurs descendants à la recherche de leurs racines, animés par une quête de sens ainsi qu'une quête personnelle, mémorielle et spirituelle.

À travers les projets portés par nos différents interlocuteurs, nous comprenons que leur souci premier est de changer l'image du vodou et de lui donner plus de visibilité sur le plan national et international. En ce sens, ils mettent à profit leurs compétences et leurs réseaux institutionnels nationaux et internationaux. Nombre d'entre eux ${ }^{16}$ ne cessent d'influencer le gouvernement au sujet de l'importance du vodou pour le peuple haïtien, méritant d'être valorisé comme patrimoine culturel du pays. Au niveau de la diaspora haïtienne, on peut également parler d'une campagne de revalorisation du vodou: conférences, publications d'articles et de livres, expositions muséales, filmsdocumentaires, organisations de voyages thématiques, sites internet et réseaux sociaux sont mobilisés dans cette perspective.

\section{BIBLIOGRAPHIE}

Arnold ANTONIN, Art naï et répression en Haïti, 45 min, couleur, 16 mm, 1976.

Arjun APPADURAI, Après le colonialisme : les conséquences culturelles de la globalisation, Payot, 2001[1996].

René BÉDARD, La représentation de l'autre dans le récit de voyage : le cas du tourisme Québécois en Haïti, mémoire de maîtrise, Université Laval, 1988.

Carlo cÉLIUS, Langage plastique et énonciation identitaire. L'invention de l'art haïtien, Les Presses de l'Université Laval, 2007.

Claudine CHASPOUL, « Confiance, respect et pédagogie ", Sites religieux et tourisme, Les Cahiers Espaces, $\mathrm{n}^{\circ}$ 96, 2007.

Yvonne Payne DANIEL, « Tourism Dance Performances: Authenticity and Creativity », Annals of Tourism Research, vol. 23, nº 4, 1996.

Joseph Ronald DAUTRUCHE, « Tourisme culturel et patrimoine remodelé : dynamique de mise en valeur du patrimoine culturel immatériel en Haïti », Ethnologies, vol. 35, n 1, 2013.

Joseph Ronald DAUTRUCHE, « Le Rara de Léogâne : entre fête traditionnelle liée au vodou et patrimoine ouvert au tourisme », Ethnologies, vol. 33, n 2, 2011.

Norluck DORANGE, The oblivion tree, film-documentaire, 2009. 
Norluc DORANGE, « Souvenance : entre le souvenir et la mémoire », Haïti en marche, vol. XXII, $\mathrm{n}^{\circ} 10$, 2008.

Daniel FABRE (dir.), Domestiquer l'histoire : Ethnologie des monuments historiques, Maisons des sciences de l'homme, 2000.

Alan GOLDBERG, « Identity and experience in Haitian voodoo shows », Annals of Tourism Research, vol. $10, \mathrm{n}^{\circ} 4,1983$.

Marie-Laurence JOCELYN-LASSÈGUE, « La culture, berceau de la renaissance haïtienne ", Le Courrier de l'UNESCO, 2010.

Christine LAURIÈRE, « D’une île à l'autre : Alfred Métraux en Haïti », Gradhiva, n 1, 2005.

Christine LAURIÈRE, « Mes amis les paysans de Marbial », Gradhiva, nº 1, 2005.

Olivier LAzZAROTTI, Patrimoine et tourisme : histoires, lieux, acteurs, enjeux, Belin, 2011.

Caroline LEGRAND, « Tourisme des racines et confrontations identitaires dans l'Irlande des migrations ", Diasporas, histoire et sociétés, $\mathrm{n}^{\circ}$ 8, 2006.

Sharon MACDONALD, « Mediating heritage. Tour guides at the former Nazi Party Rally Grounds, Nuremberg », Tourist Studies, vol. 6, n² 2, 2006.

Alfred MÉTRAUX, Le vaudou haïtien, Gallimard, 1958.

MINISTÈRE DE LA PLANIFICATION ET DE LA COOPÉRATION EXTERNE D'HAÏTI, Document de stratégie nationale pour la croissance et la réduction de la pauvreté, Ministère de la Planification et de la Coopération externe d'Haïti, 2007.

MINISTÈRE DU TOURISME, Plan Directeur Tourisme (PDT). Aménagement touristique des départements prioritaires, Ministère du Tourisme, 2008.

Pierre NORA (dir.), Les lieux de mémoires, Gallimard, 1997.

Mélaine Bertrand PODA, « Les enjeux de la patrimonialisation des lieux de mémoire à Ouidah (Bénin). Entre une expérience régionale enfouie et les perspectives mondialistes de l'UNESCO », dans Karine HÉBERT et Julien GOYETTE, Histoire et idées du patrimoine. Entre régionalisation et mondialisation, MultiMondes, 2010.

Jean PRICE-MARS, Ainsi parla l'oncle, Imprimeur II, 1998 [1928].

Graig RICHARDS (dir.), Cultural Attractions and European Tourism, CABI, 2001.

OMT, Compendium of Tourism Statistics, Organisation mondiale du tourisme, 2006.

Samuel RÉGULUS, Transmission de la prêtrise vodou. Devenir ougan ou mambo en Haïti, thèse de doctorat, Université Laval, 2012.

Laurier TURGEON, « La mémoire de la culture matérielle et la culture matérielle de la mémoire », dans Octave DEBARY et Laurier TURGEON (dir), Objets et mémoires, Maison des sciences de l'homme, 2007.

Jean-Didier URBAIN, « Tourisme de mémoire. Un travail de deuil positif », Les Cahiers Espaces, $\mathrm{n}^{\circ}$ 80, 2003.

Hécate VERGOPOULOS et Michaël BOURGATTE. « Le Ciné-tourisme comme pratique allographique », Téoros, vol. 30, $\mathrm{n}^{\circ} 1,2001$.

Nicolas VonarX, Le Vodou Haïtien : entre médecine, magie et religion, Presses de l'Université Laval, 2011. 


\section{NOTES}

1. Haïti a accueilli les premiers touristes internationaux, à proprement parler, vers la deuxième moitié des années 1940 et le pays est alors devenu l'une des plus importantes destinations touristiques des Caraïbes.

2. On constate, cependant, de nos jours, que certains pratiquants du vodou utilisent les ouvrages des ethnologues pour tracer un vèvè (symbole représentant un esprit du vodou) ou suivre les démarches d'un rituel (Régulus, 2012).

3. Alfred Métraux était alors en charge de la conduite d'enquêtes préliminaires à un projet pilote de l'Unesco consacré à l'éducation de base dans la vallée de Marbial (sud-est d'Haïti) (Laurière, 2005).

4. Les pitit kay ne résident pas forcément toute l'année à Souvenance.

5. Avec l'adoption d'une nouvelle constitution dans le pays, en 1987, consacrant la liberté religieuse, plusieurs associations regroupant des pratiquants se sont créées en vue de défendre le vodou, principalement contre d'éventuelles campagnes de dénigrement et d'éradication. Certaines de ces associations se donnent aussi pour objectif de structurer le vodou comme religion à part entière, offrant des services religieux (baptêmes, mariages, funérailles) au même titre que les églises catholiques et protestantes.

6. Nous présentons ici les projets suivant le nom d'emprunt que nous avons attribué à nos interlocuteurs. Tous ces interlocuteurs ont été rencontrés à Souvenance dans le cadre de nos terrains, en 2010 et en 2014.

7. Jean a grandi au sein de l'Église catholique et a failli devenir prêtre. Après une formation en théologie, il a fait d'autres études universitaires. Il enseigne à l'université et travaille au ministère de la Culture en Haïti. Il est initié au vodou depuis près d'une vingtaine d'années.

8. Christine est originaire des Gonaïves et son père était pratiquant du vodou à Souvenance. Elle a grandi à Port-au-Prince (la capitale d'Haïti). Loin des espaces de pratiques vodous, elle a été baptisée et a reçu sa première communion au sein de l'Église catholique. Elle a exercé le métier de journaliste en Haïti avant son départ pour les États-Unis ans les années 1970. Elle s'est initiée au vodou à la fin des années 1980 .

9. Comme c'est le cas pour beaucoup de pratiquants du vodou, Murielle a été baptisée et a reçu sa première communion au sein de l'Église catholique. Elle a également fait ses études primaires et secondaires dans une école dirigée par des religieuses catholiques. Tout en lui donnant une éducation catholique, dit-elle, ses parents pratiquaient le vodou à la maison et avaient une petite pièce réservée à des rituels. Elle a décidé de se faire initier au vodou à la trentaine, quand elle a quitté la maison de ses parents.

10. Fondé par Jaques Roumain en 1941, il offre une tribune aux intellectuels haïtiens se consacrant à l'étude du vodou et des traditions orales.

11. Cette sympathie n'a pas empêché d'autres campagnes de dénigrement et des tentatives d'éradication.

12. Un patrimoine est « difficile » dans la mesure où l'affirmation d'un sens positif de l'identité donne lieu à une lutte. Un patrimoine difficile peut également être particulièrement puissant pour éveiller de fortes émotions, être un sujet de débat public et continuer d'attirer un public potentiellement varié (Macdonald, 2006).

13. Norluck Dorange est aussi pratiquant du vodou.

14. Si le film est récent, il importe de préciser que le « rituel du retour » se déroule chaque année à Souvenance et n'a pas émergé pour répondre à la demande des visiteurs.

15. Pour une meilleure compréhension de cette idée, voir: Nora, 1997 ; Fabre, 2000 ; Urbain, 2003 ; Turgeon, 2007.

16. Certains d'entre eux cumulent plusieurs statuts: pratiquant du vodou, professeur des universités, chercheur, cadre de la fonction publique en Haïti, etc. 


\section{RÉSUMÉS}

L'emprise du vodou dans le passé touristique d'Haïti et la relance du tourisme dans ce pays, avec la culture comme marqueur principal, nous ont motivé à étudier les nouveaux rapports du couple tourisme/vodou. En s'appuyant principalement sur des recherches menées auprès de pratiquants du vodou à Souvenance (Gonaïves, Haïti), nous avançons qu'au-delà des préoccupations économiques affichées par différents gouvernements haïtiens, le tourisme est regardé par certains groupes particuliers du pays comme un forum permettant de valoriser leur communauté et de changer l'image de leur expression culturelle et religieuse.

Les nouveaux touristes que l'on rencontre dans les espaces de pratiques vodous sont surtout des Haïtiens qui ont très peu de connaissances du vodou, des visiteurs étrangers en quête de pratiques religieuses "authentiques ", des migrants haïtiens de première génération et leurs descendants à la recherche de leurs racines, animés par une quête de sens ainsi qu'une quête personnelle, mémorielle et spirituelle.

Voodoo's strong influence in the touristic past of Haiti and the revival of tourism in this country, with culture as its main icon, motivated us to study the new relationships of the binomial notions (tourism/voodoo). Based mainly on research carried out with voodoo practitioners in Souvenance (Gonaïves, Haiti), we argue that beyond the economic concerns of different Haitian governments, tourism is considered by certain groups in the country as a huge market, a golden mine and like a precious icon to enhance their community and to change and prettify the image of their cultural and religious expression.

The new tourists we meet in the spaces of voodoo practices are mostly Haitians who have very little knowledge of voodoo, foreign visitors in search of "authentic" religious practices, first generation Haitian migrants and their descendants in search of their roots, animated by a quest of meaning as well as a personal, memorial and spiritual deep quest.

\section{INDEX}

Keywords : Haiti, tourism, voodoo, migration, cultural heritage

Mots-clés : Haïti, tourisme, vaudou, vodou, migration, patrimoine culturel

\section{AUTEUR}

\section{JOSEPH RONALD DAUTRUCHE}

Enseignant-chercheur à l'Université d'État d'Haïti, Ladirep

Discipline : anthropologie

Courriel : ronaldautruche@gmail.com 